\title{
Counterpressure to the Thoracic Surface Prevents Decompression Lung Injuries in High Altitude
}

\author{
Xiaopeng Liu \\ Beihang University \\ Beijing, P.R. China \\ e-mail: liuxp01@126.com
}

\author{
Huajun Xiao \\ Institute of aviation medicine \\ Beijing, P.R. China \\ e-mail: 13621348808@163.com
}

\begin{abstract}
Objective: This study was to investigate the role of transthoracic pressure in decompression lung injuries and the effectiveness of counterpressure to the thoracic surface. Methods: Thirty-six rabbits were randomly divided into one slow decompression group(S), five rapid decompression groups (R1-R5). Counterpressure thoracic vests were used in the one rapid decompression group (R5). Rabbit mortality was recorded and the surface damages of the lungs were measured. The damage tissue was also analyzed by $H \& E$ staining and transmission electron microscope. Results: The transthoracic pressure applied to the slow decompression and 5 rapid decompression groups were $0 \mathrm{kPa}(\mathrm{S}), 7.52 \mathrm{kPa}$ (R1), 9.77 kPa (R2), $11.89 \mathrm{kPa}(\mathrm{R3}), 13.62 \mathrm{kPa}$ (R4) and $13.07 \mathrm{kPa}$ (R5), respectively. There were no obvious lung injuries in $\mathrm{S}$ group. Minor injuries were observed in R1 group. With the increase of transthoracic pressure, the degree of severity of pulmonary congestion and edema, alveolar hemorrhage, and lung rupture increased. The use of counterpressure thoracic vests prevented the lung injuries caused by high transthoracic pressure. Conclusions: Transthoracic pressure plays an important role in decompression lung injuries. The decompression lung injuries worsen with the increase of transthoracic pressure. Counterpressure thoracic vests can prevent the decompression lung injuries.
\end{abstract}

Keywords-rapid decompression; lung injuries; transthoracic pressure; animal model; protective vest

\section{INTRODUCTION}

Most commercial aircraft and military combat aircraft are equipped with pressurized cabin, which can solve many physiological problems associated with high altitude flight, such as hypoxia[1], ADS[2,3,4] and mark a significant advance in passengers comfort and safety[5]. However, it also created a new potential hazard, the loss of cabin pressurization due to mechanical failure, structural damage, battle damage, etc. $[2,3,4,5,6,7,8]$. Over the 20 years (1962-1982), there have been 47 cases of serious loss of cabin pressure in Canada Force ejection seat equipped aircraft, in which 29 incidents were massive/explosive loss of pressure[7]. During 1969 to 1990, there were 205 reported cases of loss of cabin pressure in US Naval aircraft. Of these cases, $117(57.07 \%)$ were considered slow and $88(42.93 \%)$ were rapid decompressions[6].
The incidence of pressurized cabin decompression are not high on military and civilian aircraft, but the risk to pilots are definite, including expansion of gases in closed and semi-closed cavities, hypoxia, decompression sickness, etc $[7,9,10]$. The lungs are potentially the most vulnerable organ due to the delicate nature of the pulmonary tissue and large volume of air in the lungs during rapid decompression [11]. Slow decompression do not cause an obvious lung injury, but the rapid decompression do cause lung injuries in different degrees[12]. Many studies focus on investigating the lung injuries under different decompression speed or pressure differential[13,10,14]. Whenever a rapid decompression is faster than the inherent capability of the lungs to decompress, a transient positive pressure will temporarily build up in the lungs [15]. The maximal transient positive pressure is peak transthoracic pressures. The transthoracic pressure may vary with different people and/or with different oxygen supply system in the same decompression speed or pressure. Therefore, it is very important to investigate the role of transthoracic pressures, in particular, peak transthoracic pressures in rapid decompression lung injuries. Furthermore, it is also important to investigate the possible countermeasure to prevent these injuries.

The purpose of the current research is to evaluate the lung injuries in different peak transthoracic pressures at same decompression speed and the effectiveness of counterpressure to the thoracic surface on lung protection.

\section{METHODS}

\section{A. Animals}

This study was conducted according to the Guide for the Care and Use of Laboratory Animals of Chinese Academy of Sciences. There were $36 \mathrm{New}$ Zealand Rabbits (10wk old) in this study, which weighed $2,680 \pm 193$ g. All rabbits had free access to a standard water and chow. Those rabbits were randomly assigned to 6 groups, one slow decompression group (S) and five rapid decompression groups (R1-R5), and each group had 6 rabbits.

\section{B. Equipments}

The altitude control system consists of experimental cabin, hypobaric cylinder, decompression device, pressure sensor and resistance adjusting device (Fig.1). The 
experimental cabin and hypobaric cylinder were used to simulate hypobaric environment of altitude. The decompression device was used to control the speed of decompression of experimental cabin. The pressure sensor was used to measure the pressure in rabbits lungs. The resistance adjusting device was used to control the speed of air outflow out of rabbits lungs by changing the diameter of vents of air outflow during decompression.

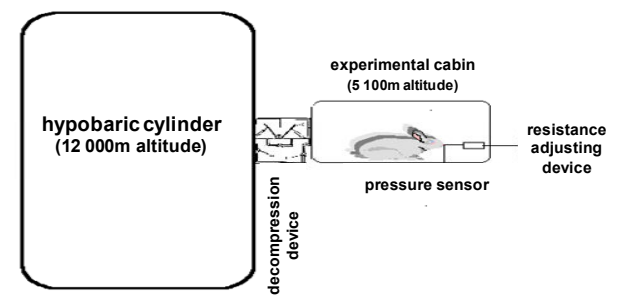

Figure 1. Schematic representation of the altitude control system

\section{Procedure}

All rabbits were free access to water and food but were starved for $24 \mathrm{~h}$ prior to the experiment. Rabbits were fixed on operating table and a $\mathrm{Y}$ tracheotomy connector was inserted to the rabbit trachea under local anesthesia with $1 \%$ procaine. The two ends of the $\mathrm{Y}$ tracheotomy connector were connected to a pressure sensor and a resistance adjusting device, through which the target peak transthoracic pressures were achieved, respectively. 10 min after surgery, rabbit was placed into the experimental cabin (Fig. 1) which is connected with hypobaric cylinder by a decompression device. The altitude of cabin ascended to $5,100 \mathrm{~m}$ at the rate of $15 \mathrm{~m} / \mathrm{s}$ and the rabbit stayed at $5100 \mathrm{~m}$ for $10 \mathrm{~min}$ before decompression.

In slow decompression group, the cabin pressure decompressed from 5,100 m altitude to $12,000 \mathrm{~m}$ altitude within 69 seconds. In rapid decompression groups, the cabin pressure decompressed from $5,100 \mathrm{~m}$ altitude to $12,000 \mathrm{~m}$ altitude within 0.3 second. The diameter of vents of resistance adjusting device was 5.0, 4.0, 3.0, 2.0 and 2.0 $\mathrm{mm}$ in $\mathrm{R} 1$ to $\mathrm{R} 5$ group, respectively. In the R5 group, a protective vest was given to the rabbit as the countermeasure. After decompression, the altitude of experimental cabin descended to ground altitude $(43 \mathrm{~m}$, altitude of Beijing, China) in $60 \mathrm{~s}$ in order to minimize the effects of hypoxia.

\section{Measurements}

Peak transthoracic pressure was measured. Rabbit mortalities were recorded, the surface damages of the lungs were measured. Lung injury severity scores were used to assess the degree of injuries in lungs by using the scoring method in our laboratory. In brief, no injury was 0 ; mild injury was 1 point, which was defined as dot hemorrhage and hemorrhage area less than $10 \%$ of lung surface; medium injury was 2 points, which was defined as dot hemorrhage or splinter hemorrhage and hemorrhage area less than $50 \%$ of lung surface; severe injury was 3 point, which is defined as splinter hemorrhage and hemorrhage area about $50 \%$ of lung surface; extremely severe injury was 4 points, which was defined as many splinter hemorrhage and hemorrhage area more than $50 \%$ of lung surface or hemorrhagic bubble in trachea.
The tissue between the bleeding and non-bleeding area of the left lung was used for pathological examination. The lung tissues of 1.0 cubic $\mathrm{cm}$ were fixed with $10 \%$ formaldehyde solution. Fixed lungs were embedded in paraffin and sectioned by standard methods. For histological analysis, 3-5 $\mu \mathrm{m}$ thick sections were cut and stained with hematoxylin and eosin(H\&E) and examined under optical microscope. The lung tissues of $0.1 \mathrm{~cm} \times 0.1$ $\mathrm{cm} \times 0.1 \mathrm{~cm}$ were fixed with $2.5 \%$ glutaraldehyde solution, which were examined under transmission electron microscope by standard method.

\section{E. Data Analyses}

One-way ANOVA with protected post hoc Fisher's LSD tests was used to compare difference between the six groups. Bivariate correlation of pearson correlation coefficients was used to analyze the relation between diameter of vents and peak transthoracic pressure. Data were expressed as mean $\pm \mathrm{SD}$. Statistical significance was considered at $\mathrm{P} \leqslant 0.05$.

\section{RESULTS}

\section{A. Animal model}

Peak transthoracic pressure did not appear in S group. Peak transthoracic pressure was observed from $7.52 \mathrm{kPa}$ to $13.62 \mathrm{kPa}$ and the differences were significant $(\mathrm{F}=103.874$, $\mathrm{P}<0.001$ ) in $\mathrm{R} 1-\mathrm{R} 5$ groups (Table I). The Peak transthoracic pressure of $\mathrm{R} 1$ group rabbits was smaller $(\mathrm{P}<0.001)$ than that in $\mathrm{R} 2-\mathrm{R} 5$. The Peak transthoracic pressure of $\mathrm{R} 5$ group rabbits was not different $(\mathrm{P}=0.133)$ from R4 group but greater than in R1-R3.

There are significant correlation statistically between peak transthoracic pressure $(\mathrm{Pt})$ and vents diameter of resistance adjusting device $(\mathrm{V})$ in $\mathrm{R} 1$ to $\mathrm{R} 4$, and the standardized regression coefficient Beta was 0.968 $(\mathrm{P}<0.01)$. The regression equation was that $\mathrm{Pt}=17.845$ 2.042V (Fig. 2).

TABLE I. VENTS DIAMETER AND PEAK TRANSTHORACIC PRESSURE IN S AND R1-5 GROUPS

\begin{tabular}{|c|c|c|c|}
\hline Groups & $\begin{array}{c}\text { Diameter of } \\
\text { Vents }(\mathbf{m m})\end{array}$ & $\begin{array}{c}\text { Peak Transthoracic } \\
\text { Pressure } \\
(\boldsymbol{k P a})\end{array}$ & $\begin{array}{c}\text { Protective } \\
\text { Vest }\end{array}$ \\
\hline S & --- & 0 & No \\
\hline R1 & 5.0 & $7.52 \pm 0.76 \dagger \dagger$ & No \\
\hline R2 & 4.0 & $9.77 \pm 0.30^{*} \dagger \dagger$ & No \\
\hline R3 & 3.0 & $11.88 \pm 0.78^{*} \dagger$ & No \\
\hline R4 & 2.0 & $13.62 \pm 0.56^{*}$ & No \\
\hline R5 & 2.0 & $13.08 \pm 0.49^{*}$ & Yes \\
\hline
\end{tabular}

Data were mean $\pm \mathrm{SD}$, slow decompression group (s), rapid decompression groups with different rabbits. ${ }^{*} \mathrm{P}<0.001$, compared with $\mathrm{R} 1 ; \uparrow \mathrm{P}<0.01$, $\dagger$ † $\mathrm{P}<0.001$, compared with $\mathrm{R} 5$. 


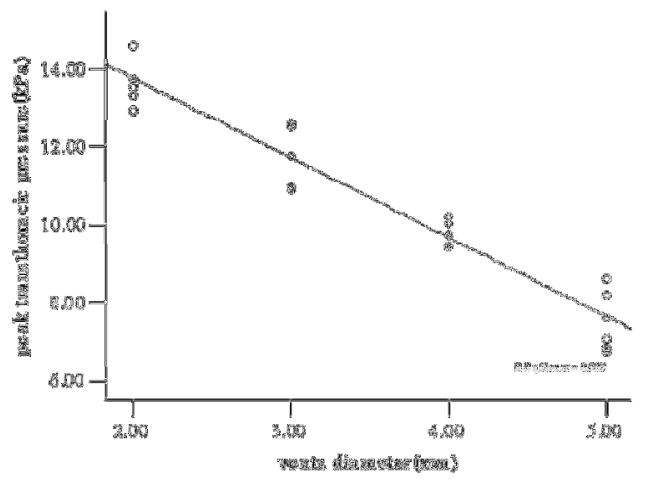

Figure 2. Negative correlation between transthoracic peak pressure and vents diameter in R1-4 groups

\section{B. Mortality rate and lung injury severity scores}

There was no mortality in $\mathrm{S}$ group, R1 and R2 group. Mortality appeared when peak transthoracic pressure reached to $11.89 \mathrm{kPa}$ and the mortality rate increased with the increase in peak transthoracic pressure (Table II). However, wearing protective vest prevented mortality even the transthoracic pressure was high as $13.07 \mathrm{kPa}$. Lung injuries did not appear in $\mathrm{S}$ group and injury severity score significantly increased in all rapid decompression groups; the higher the peak transthoracic pressure was, the larger the injury severity score was, even the rapid decompression speed was same $(\mathrm{F}=22.222, \mathrm{P}<0.001)$. It suggests that the transthoracic pressure plays great role in rapid decompression caused lung injuries during rapid decompression. The injury severity score in R5 group was not significantly different when compared with that in R1 $(\mathrm{P}=0.302)$ and $\mathrm{R} 2(\mathrm{P}=0.302)$ group.

TABLE II. THE MORTALITIES AND LUNG INJURY SEVERITY SCORES IN S AND R1-5 GROUPS

\begin{tabular}{|c|c|c|}
\hline Groups & Death Number & Lung Injury Severity Scores \\
\hline S & 0 & $0.00 \pm 0.00$ \\
\hline R1 & 0 & $0.83 \pm 0.41$ \\
\hline R2 & 0 & $1.50 \pm 0.55 \dagger$ \\
\hline R3 & 1 & $2.17 \pm 0.41 * \dagger \dagger$ \\
\hline R4 & 3 & $3.50 \pm 0.84 * * \dagger \dagger$ \\
\hline R5 & 0 & $1.17 \pm 0.41$ \\
\hline
\end{tabular}

Mean $\pm \mathrm{SD}$ of Lung Injury Severity Scores in six groups $(\mathrm{N}=6)$. Significantly different from $\mathrm{R} 1$ $\dagger \mathrm{P}<0.05$, $\dagger \dagger \mathrm{P}<0.001$. Significantly different from $\mathrm{R} 5$ : $* \mathrm{P}<0.01,{ }^{* *} \mathrm{P}<0.001$.

\section{The distribution and severities of lung injuries under different peak transthoracic pressure at gross examination.}

There was no obvious hemorrhage, edema or other injuries on the surface of lungs in $\mathrm{S}$ group. Lung injuries worsened when the peak transthoracic pressure increased in R group, which included four degrees, mild, medium, severe and extremely severe according to the extent of injury. Mild injuries were hyperemia, edema, dot hemorrhage or patchy hemorrhage. Medium injuries were splinter hemorrhage. Severe injuries were massive hemorrhage with different extent of edema, which often involved deep lung tissue, red bubbles in tracheas of some rabbits, and even "rib imprint" congestion and/or hemorrhages. Extremely severe injuries were diffuse hemorrhage and even alveolus rupture. In R1 group only had mild injuries; in R2 group, mild and medium injuries were observed; in R3 group, most part of lung has several severe injuries and mixed with some mild injuries. All part of lungs in R4 group had extremely injuries. However, only mild lung injuries was observed in R5 group which had protective vest (Fig. 3).

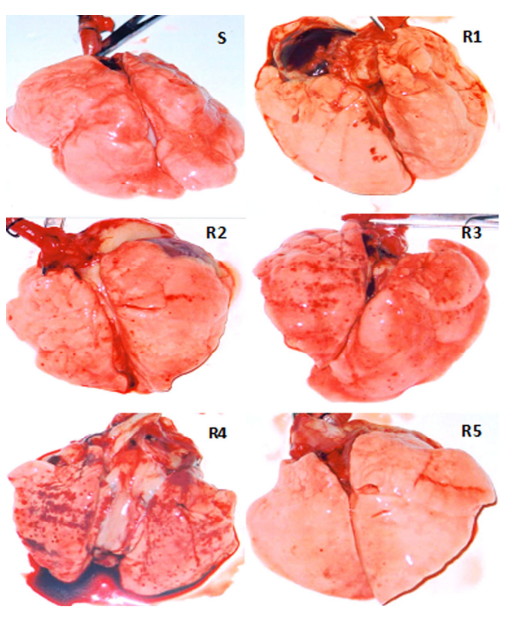

Figure 3. Gross distribution and amount of lung injuries

\section{The lung damages under H\&E staining}

In $\mathrm{S}$ group, the alveolar ducts and the alveolar sacs were distinct and intact; alveolar spaces were inerratic; alveolar septum were clear; capillary vessels, arterioles, venules and bronchiole were normal.

In R1 group, arterioles, venules and bronchiole were basically normal; some alveolar ducts, alveolar sacs and alveoli expanded; alveolar septum widened with slight hyperemia and edema; capillary vessels slightly expanded.

In R2 group, alveolar ducts, alveolar sacs and alveoli expanded; some alveolar walls were broken; alveolar septum widened with splinter hyperemia and edema; capillary vessels expanded and congested; lung tissues around the arterioles and bronchiole congested slightly; macrophages and blood cell appeared in some alveolar spaces.

In R3 group, alveolar ducts, alveolar sacs were damaged; lots of alveolar walls were broken and became pneumatocele; alveolar tissues were compressed and little lung detelectasis appeared around the pneumatocele; macrophages and blood cell appeared in alveolar spaces; alveolar septum widened with splinter hyperemia and edema; capillary vessels expanded and congested; lung tissues around the arterioles and bronchiole congested.

In R4 group, alveolar ducts, alveolar sacs were badly damaged; lots of alveolar walls were broken and became pneumatocele; alveolar septum widened with splinter hyperemia and edema; capillary vessels expanded and 
congested; alveolar spaces were full of macrophages and blood cells.

In R5 group, lung injuries were similar to that in R1 group.

In brief, the lung damages were getting serious with the increase of the peak transthoracic pressure. Wearing protective vest could prevent this damage in the lungs (Fig. 4).

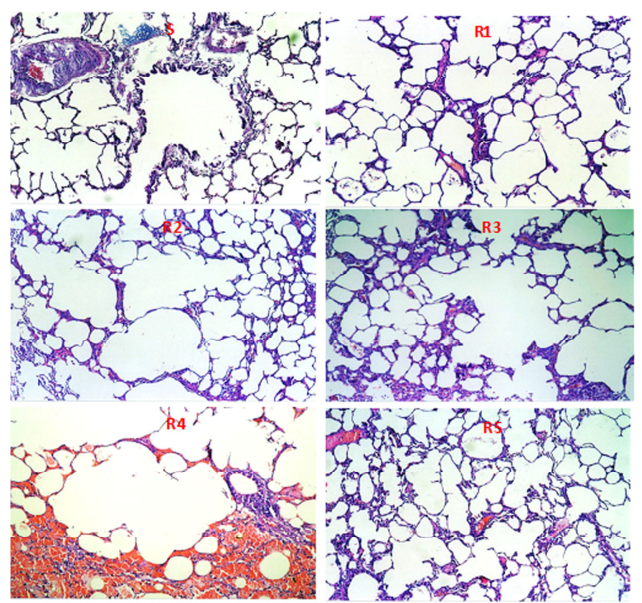

Figure 4. Histological lung injuries $(H \& E$ staining, $\times 100)$ The alveoli were generally intact, and architectural integrity of the alveoli was well preserved in S group. Enlargement of alveolar spaces were observed in $\mathrm{R} 1$ group and some alveolar tears were observed in R2 group. Distensions of ruptured alveolar septa with consecutive enlargement of alveolar spaces and intraalveolar hemorrhages were clearly shown in R3 and R4 groups. The protective vest prevented the major damage to the lung in R4 group.

\section{E. Electron micrographs of lung in rabbits under different peak transthoracic pressures}

There were no ultrastructural pathological changes determined in S group. Type I and II pneumocyte were clear; alveolar septum were normal; membrane structures were clear and the karyon chromatins were welldistributed. There were a few endoplasmic reticulums and other cellular organelles and well-distributed in Type I pneumocyte; In Type II alveolus cells, there were rough surfaced endoplasmic reticulum (RER), Golgi complexes, rich free ribosome and many multivesicular bodies and lamellar bodies; there were short microvilli on the surface of Type II alveolus cells; the connection between Type I and II pneumocyte were normal; the mesenchymal cells, the vascular endothelial cells and the structure of air-blood barriers (ABB)were normal.

In R1 group, slight edema appeared in Type I pneumocyte; the rough surfaced endoplasmic reticulums (RE) in Type II pneumocyte were slightly dilatate; the capillary vessels on the alveolar wall were slightly expanded and congested.

In R2 group, slight edema appeared in Type I pneumocyte; the rough surfaced endoplasmic reticulums (RE) were slightly dilatate and mitochondria were swelling in Type II pneumocyte; the capillary vessels on the alveolar wall and the vascular endothelial cells (VEC) were slightly swelling.

In R3 group, edema appeared in Type I pneumocyte; there were intense intracellular vacuoles, dilatate rough surfaced endoplasmic reticulums (RE) and swelling mitochondria in Type II pneumocyte; The capillary vessels on the alveolar wall and the vascular endothelial cells (VEC) were swelling; the structure of the air-blood barriers (ABB) were damaged and there were blood cells and proteins in the alveolar spaces.

For R4 group, membrane structure of Type I and Type II pneumocyte were unclear; the connection between pneumocyte was loose; edema appeared in Type I pneumocyte; there were dilated rough surfaced endoplasmic reticulums (RER) and swelling mitochondria and unclear lamellar bodies; the microvilli were detached from the surface of type II pneumocyte; The capillary vessels on the alveolar wall and the vascular endothelial cells (VEC) were swelling; the structure of the air-blood barriers $(\mathrm{ABB})$ were damaged; the alveolar spaces were full of leaked red cells and seeped macrophages.

For R5 group, slight edema appeared in Type I pneumocyte; the rough surfaced endoplasmic reticulums (RER) were slightly dilatate and mitochondria were slightly swelling in Type II pneumocyte; The capillary vessels on the alveolar wall and the vascular endothelial cells (VEC) were swelling. The pathological changes were similar to that in R1 group (Fig. 5).

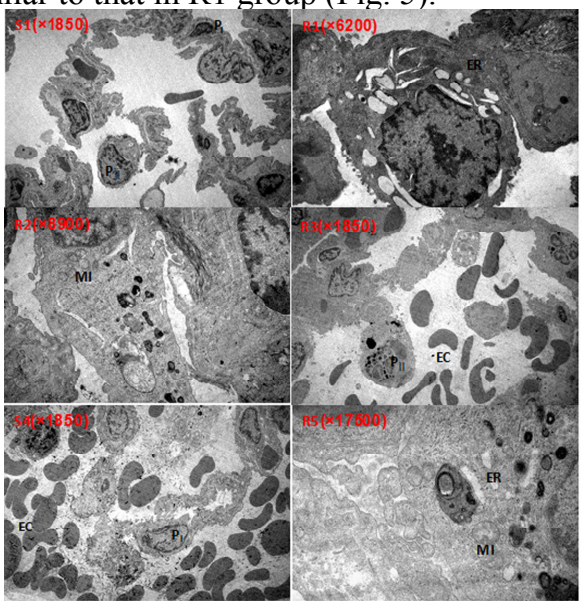

Figure 5. Microstructural lung injuries (TEM) There were no ultrastructural pathological changes determined in $\mathrm{S}$ group. There were dilatation in endoplasmic reticulum in $\mathrm{R} 1$ group. Swelling of mitochondria was observed in R2 group. In R3 group, there were intense intracellular vacuoles in type II pneumocyte. Blood cells circulated through capillaries. The changes in R4 group were similar to R3 group but got worse, such as edema observed in type I pneumocyte. In R5, there was only slight dilatation in endoplasmic reticulum and swelling of mitochondria. PI, Type I pneumocyte; PII, Type I pneumocyte; ER, endoplasmic reticulum; MI, mitochondria; EC, erythrocyte

\section{DISCUSSION}

Studies on lung injury caused by rapid decompression have been lasted more than 70 years. As early as 1936, Armstrong started the research on the effect of rapid decompression on animals. For several decades, the aviation medical workers of different countries have conducted several studies and believed that, in case of rapid decompression, the peak transthoracic pressures reaching certain pressure threshold is the basic cause for lung injury[15,16,17]. Those studies suggested that the transthoracic pressure may depend on the speed of decompression and the fraction differential 
(differential/initial pressure) in decompression regardless of the initial cabin pressure, and the mean flow resistance offered by the human airways appears to be considerably greater in rapid decompression than during spontaneous, quiet breathing. However, those studies did not investigate the certain pressure threshold that could lead to lung injuries and neglected the openness extent of glottis on air flow assistance which could affects the decompression transthoracic pressure. Additionally, there were also different opinions about the effects of counterpressure to the thoracic surface on decompression lung injuries. Therefore, in this study, we adjusted the ventilator size to achieve different degrees of transthoracic pressure to explore pressure threshold of lung injuries and whether the counterpressure provided by protective vest could counter lung injuries.

This study was the first one to use controlled peak transthoracic pressures animal model. Firstly, the designed peak transthoracic pressures were achieved by changing the resistance of expanded air outflow in lungs during decompression. The vents of the resistance adjusting device, which was connected to Y tracheotomy connector, were circular and the diameter of vents was changed from 1 to $10 \mathrm{~mm}$. In the experiment, the vent diameter of $\mathrm{R} 1 \sim$ R4 was 5.0, 4.0, 3.0 and $2.0 \mathrm{~mm}$, and the corresponding peak transthoracic pressures were $7.52 \mathrm{kPa}, 9.77 \mathrm{kPa}$, $11.89 \mathrm{kPa}$ and $13.62 \mathrm{kPa}$, which were significantly different. Secondly, the peak transthoracic pressures were recorded with a pressure sensor which was fixed at $Y$ tracheotomy connector introduced directly into each rabbit tracheas. The transthoracic pressures measured by this way were accurate than other methods, which pressure sensors were fixed in right atrium[16] or thoracic esophagus[17]. Thirdly, 1\% procaine was used in all experiments for local anesthesia involving surgery (tracheal intubation) instead of general anesthesia, so the rabbits were almost in a normal condition and their thorax and midriff could work in protection against lung injuries. Finally, the closure or openness of glottis could not affect the transthoracic pressure due to tracheal intubation. The glottis closure increased the outflow resistance of air in lungs during rapid decompression, vice versa. After tracheotomy surgery, the air outflowed from lungs by $\mathrm{Y}$ tracheotomy connector instead of glottis. Therefore, it was insured that the air flow resistances were similar in same group, which was beneficial to control the transthoracic pressure.

The expanding gas in the lung of the animals of the slow group had sufficient time to escape from the respiratory tract during slow decompression, which did not result in any transthoracic pressures. Therefore, there were also no lung injuries in the slow group, which was confirmed by lung injury severity score and the results of H\&E staining and transmission electron microscope. When rapid decompression happened, the air in the experimental cabin expanded and escaped much faster than air in lungs that caused transthoracic pressure. When the peak transthoracic pressure was below $9.77 \mathrm{kPa}$, the rabbit lung only suffered from slight or intermediate injuries. The lung injury severity had gradually increased with the increase of peak transthoracic pressure. While it reached to $13.62 \mathrm{kPa}$, the rabbit lungs had experienced severe or extremely severe injuries and lung injury severity score rose from 0.83 to 3.5 .
Rapid decompression had caused remarkable injuries on rabbit lung, especially in Group R3 and Group R4, whose peak transthoracic pressures were above $11.89 \mathrm{kPa}$, including lung edema, lung hemorrhage and even rupture. Transthoracic pressures resulted in lung expansion and alveolar wall stretching, which would increase the permeability of the interalveolar membrane outer layer to solute. Once the improved permeability exceeds the critical value of some small solute, according to the Starling Law[18), those small solute can enter the alveolus, which will raise osmotic pressure of alveolus and then bring the tissue fluid into the alveolus. Birgham (1978), Effros (1982), Egan (1976), Nicolaysen and Hauge (1982) have already confirmed that such permeability change on the exodermis may be the cause for alveolus edema [18]. As the compliance of hundreds of millions of alveoli and the resistance of the small air ducts between these alveoli differ, expansion extent of alveoli varies during rapid decompression. Stretching or overexpansion of alveoli would lead to alveolar walls fragmentized [19]. The peak transthoracic pressures in Group R3 and Group R4 were so high to bruise lungs against the resistant thorax. The strength of impaction mainly depended on the peak transthoracic pressure and lungs injuries, such as hemorrhage and edema, were observed in the surface of lungs while the force exceeded the threshold value the lung tissue can stand[12,20,21,22]. Congestive or bleeding impressions could be found in Fig.3.

In Group R3 and Group R4, very severe ultrastructure changes occurred, which were vacuolate Type II pneumocyte, endoplasmic reticulum expansion, mitochondria swelling, and the capillary vessels expansion and hyperemia with permeability increased. These changes in Group R4 got worse. The connection between cells got loose; the air-blood barrier structure was destroyed; and the alveolar were full of leaked red cells and seeped macrophagocytes. Such severe injuries in the ultrastructure of the lungs of the rabbits were most likely the pathological basis for their lung functional disorder, which could result in acute respiratory failure and the death of rabbits[23].

It is generally believed that the ability to endure rapid decompression is lie on the ability of lung to endure peak transthoracic pressure. The higher peak transthoracic pressure is, the severer lung injuries are. The lungs in the slow compression group had the normal structure. The lungs in rapid decompression group with $7.52 \mathrm{kPa}$ and $9.77 \mathrm{kPa}$ peak transthoracic pressure had light changes, which could not seriously reduce the respiratory function and could recover soon if secondary infection of lung did not happen. The results indicated that $9.77 \mathrm{kPa}$ peak transthoracic pressure could be the threshold of rabbits lung injuries.

There were different opinions whether the counterpressure to the thoracic surface was beneficial to lung protection during rapid decompression. Whitehorn's work had shown that the addition of a binding or a suit to the thoracic cage which restricted chest expansion caused more pulmonary damage than that occurring in an unrestricted animal [24]. However, Barron believed that wearing waistcoat can minimize the possibility of lung injury [25] and Rosenbaum also thought that wearing full bladder suit could alleviate pulmonary damage[14]. In this 
study, rabbits of group R5 were protected with waistcoats, which provided counterpressure to thoracic and abdominal surface to restrict the thorax from overexpanding during rapid decompression. The peak transthoracic pressures in group R5 were similar to that in group R4, but injury severity score was only one-third of that in group R4. The peak transthoracic pressures in group R5 were far higher than that in group R2, but lung injury severity in gross and pathological inspection in both group were not significant different and light, which could recover soon. In our daily life, we have similar experience, for example, cough, weight lifting and Valsalva action, etc. can increase transthoracic pressure to $20.0-40.0 \mathrm{kPa}(150-300 \mathrm{mmHg})$, which do not cause lung injuries due to contracted pectoral and abdominal muscles to limit the overexpansion of thorax[11]. In this experiment, The use of counterpressure thoracic vests decreased the lung injuries caused by 13.07 $\mathrm{kPa}$ to be similar as that observed in $9.77 \mathrm{kPa}$ group. Counterpressure thoracic vests can minimize the decompression lung injuries. It was concluded that the threshold of rabbits lung rapid decompression injuries may rise from $9.77 \mathrm{kPa}$ peak transthoracic pressure to 13.07 $\mathrm{kPa}$ after rabbits vests provided counterpressure to the thoracic surface to restrict thorax overexpansion during rapid decompression. However, in this study, as we did not have a long-time observation over the injured rabbits, their recovery and endurance limit against rapid decompression still need further investigations in the future.

In brief, peak transthoracic pressure was the major factor which causes lung injuries during rapid decompression. Wearing protective vest can prevent the lung damages, which might come from restricting the expansion of the lung.

\section{ACKNOWLEDGMENT}

This study was kindly supported by institute of aviation medicine, China.

\section{REFERENCES}

[1] Davis JR, Johnson R, Stepanek J, et al. Respiratory physiology and protection against hypoxia// Pickard JS, Gradwell DP. Fundamentals of Aerospace Medicine, 4th ed., Philadephia:Lippincott Williams \& Wilkins, 2008, pp.35-37.

[2] Davis JR, Johnson R, Stepanek J, et al. Physiology of decompressive stress// Stepanek J, Webb JT. Fundamentals of Aerospace Medicine, 4th ed., Philadephia:Lippincott Williams \& Wilkins, 2008, pp.64-65.

[3] Liu Xiaopeng. "Physiological research progress on pilot's high altitude compensating suit," Chin J Aeros Med, vol. 22, Feb. 2011, pp.56-59, DOI:10.3760/cma.j.issn.10076239.2011.01.020.

[4] Xiao Hunjun. Physiology of aviation oxygen protective equipment, Beijing: Military Medical Science Press, 2005, pp.189-274.

[5] Fang HS, Chang YN. "Application of the compartmentalization/airlock concept to aircraft and tolerance of lung to rapid decompression," Aviat Space Environ Med, vol. 55, Nov. 1984, pp. 1015-1019, PMID:6508681.

[6] Bason R, Yacavone DW. "Loss of cabin pressurization in U.S.Naval aircraft:1969-90,” Aviat Space Environ Med, vol. 63, May. 1992, pp. 341-345, PMID:1599378.

[7] Brooks CJ. "Loss of cabin pressure in Canadian Forces election seat aircraft,1962-1982," Aviat Space Environ Med, vol. 55, Dec. 1984, pp. 1154-1163, PMID:6517823.
[8] Johnston MJ. "Loss of cabin pressure in a military transport: a mass casualty with decompression illnesses." Aviat Space Environ Med, vol. 79, Apr. 2008, pp. 429-432, DOI:10.3357/ASEM.2153.2008.

[9] Connolly DM, D'Oyly TJ, McGown AS, Lee VM. "Lung volumes, pulmonary ventilation, and hypoxia following rapid decompression to $60,000 \mathrm{ft}(18,288 \mathrm{~m})$," Aviat Space Environ Med, vol.84, Jun. 2013, pp. 551-559, DOI:10.3357/ASEM.3533.2013.

[10] Files DS, Webb JT, Pilmanis AA. "Depressurization in military aircraft: rates, rapidity, and health effects for 1055 incidents," Aviat Space Environ Med, vol. 76, Jun. 2005, pp. 523-9, PMID:15945394.

[11] Yu Zhibin. "Aerospace physiology," $\mathrm{Xi}$ an: Fourth Military Medical University Press, 2008, pp.44-49.

[12] Chen Haibin, Wang Zhengguo. "Injury action on rabbit lung by explosive decompression," Chin J Trauma, vol. 16, Feb. 2000, pp. 109-111.

[13] Close P, Ireland R. "Effect of certain variations in the physiologic state on tolerance to explosive decompression," Aerosp Med, vol. 32, Nov. 1961, pp. 1050-1060, PMID:13879987.

[14] Rosenbaum DA. "Explosive decompression studies with animals wearing full bladder suit and helmet," J Aviat Med. vol. 30, Apr. 1959, pp. 251-257, PMID:13641179.

[15] Topliff ED. "Mechanism of lung damage in explosive decompression," Aviat Space Environ Med, vol. 47, May. 1976, pp. 517-522, PMID:1275842.

[16] Boyle J. "Theoretical trans-respiratory pressure during rapid decompression: I Model experiment. II Animal experiments," Aerospace Med, vol. 44, Feb. 1973, pp. 153162, PMID:4687830,4687831.

[17] Luft UC, Bancraft RW. "Transthoracic pressure in man during rapid decompression," J Aviation Med, vol. 27, Jun. 1956, pp. 208-220, PMID:13319240.

[18] Yuan-Cheng Fung. Biomechanics. Translator: Deng Shanxi. Chengdu(China): Sichuan Educational Press. 1993. 608614.

[19] Zhu Xiangqi, Cheng Guoguang. "Pulmonary injury in animals induced by compression-rapid decompression," Chin J Appl physiol, vol. 13, Mar. 1997, pp. 280, PMID:10074290.

[20] Chen Haibin, Wang Zhengguo. "Role of overtension effect in blast lung injury," Acta Academiae Medicinae Tertiae, vol.22, Feb. 2000, pp.106-108.

[21] Hitchcock FA. "Physiological and pathological effects of explosive decompression," J Aviat Med, vol. 25, Dec. 1954, pp. 578-86, PMID:13221521.

[22] Zhang Junkui, Wang Zhengguo, Yang Zhihuan, et al. "Pathological changes of underpressure-induced lung injury in rats," Acta Academiae Medicinae Militaris Tertiae, vol. 16, Jan. 1994, pp. 10-12.

[23] Wang Zhengguo. Blast Injury. Beijing: People'S Military Medical Press, 1983, pp.142-150.

[24] Whitehorn WV, Lein A, Hitchcock FA. "The effect of binding of abdomen and thorax on pulmonary lesions produced by explosive decompression," J Aviation Med, vol. 18, Feb.1947, pp.102-104, PMID:20287797.

[25] Barron CI, Cook TJ. "Effects of variable decompressions to 45000 feet," Aerosp Med, vol. 36, May. 1965, pp. 425-430. 